\title{
A NOTE ON EXTENDED AMBIGUOUS POINTS
}

\section{J.L. STEBBINS}

Let $f$ be an arbitrary function from the open unit disk $D$ of the complex plane into the Riemann sphere $S$. If $p$ is any point on the unit circle $C, C(f, p)$ is the set of all points $w$ such that there exists in $D$ a sequence of points $\left\{z_{j}\right\}$ such that $z_{j} \rightarrow p$ and $f\left(z_{j}\right) \rightarrow w . \quad C_{\Delta}(f, p)$ is defined in the same way, but the sequence $\left\{z_{j}\right\}$ is restricted to $\Delta \subset D$. If $\alpha$ and $\beta$ are two arcs in $D$ terminating at $p$ and $C_{\alpha}(f, p) \cap C_{\beta}(f, p)=\Phi, \quad p$ is called an ambiguous point for $f$.

Mathews (3, p. 138) defined an extended ambiguous point for $f$ in the following way: Let $\alpha$ be an arc in $\bar{D}-\{p\}$ terminating at $p . E C_{\alpha}(f, p)=$ $\cap \overline{U C(f, q)}$ where the intersection is taken over all neighborhoods $N$ of $p$ and the union over all $q$ on $\alpha \cap N, q \neq p$, and $C(f, q)=\{f(q)\}$ if $q \in D$.

Bagemihl (1) proved that an arbitrary function from $D$ into $S$ can have at most countably many ambiguous points. Mathews (3, p. 138, proof of Theorem 1) has proved that a countinuous function from $D$ into $S$ can have at most countably many extended ambiguous points. This proof does not hold if $f$ is not continuous (the statement that $C_{B L}(g, 1)$ is contained in either $E C_{\alpha}(f, p)$ or $E C_{\beta}(f, p)$ only holds if $f$ is continuous). The theorem, however, does hold.

TheOREM (Matherws): If $f$ is an arbitrary function from $D$ into $S$ and if a point $p$ on $C$ is an extended ambiguous point for $f$, then $p$ is an ambiguous point for $f$.

Proof. Let $\alpha$ be any arc in $\bar{D}-\{p\}$ such that $\alpha$ tends to $p$. As Mathews' indicates, it is sufficient to find an arc $\alpha^{\prime} \subset D$ and tending to $p$ such that $C_{\alpha^{\prime}}(f, p) \subset E C_{\alpha}(f, p)$. Use the points $q \in \alpha \cap C$ and the method of Gross (2, p. 249) to construct a "wedge" $Z_{1}$ in $D$ such that for every sequence of points $\left\{z_{k}\right\}$ in $Z_{1}$ tending to $p,\left\{f\left(z_{k}\right)\right\}$ has limit points only in $\cap \overline{U C(f, q)}$

Received September 4. 1970. 
where $q \in \alpha \cap C, q \neq p$, and the intersection is taken over all neighborhoods of $p$.

In general the set $Z_{1}$ will not be connected since $\alpha \cap C$ will not always contain a terminal part of $\alpha$. However, $\alpha \cap D$ may be used to join the components of $Z_{1}$ so that $(\alpha \cap D) \cup Z_{1}$ will contain an $\operatorname{arc} \alpha^{\prime}$ tending to $p$. It is clear that $C_{\alpha \prime}(f, p) \subset E C_{\alpha}(f, p)$, and the theorem follows.

\section{REFERENCES}

[1] Bagemihl, F., Curvilinear cluster sets of arbitrary functions. Proc. Nat. Acad. Sci. U.S.A. 41, 379-382 (1955).

[2] Gross, W., Zum Verhalten analytischer Funktionen in der Umgebung singulärer Stellen. Math. Z. 2, 243-294 (1918).

[ 3 ] Mathews, H.T., A note on Bagemihl's ambiguous point theorem. Math. Z. 90, 138-139 (1965).

University of Wisconsin-Milwaukee 